assessed the patient was also categorised as trainee, GP or locum. Results: $\mathrm{n}=15$. Overall $13 \%$ referrals were appropriate, $33 \%$ inappropriate and $53 \%$ missed. $100 \%$ trainee referrals were inappropriate, $80 \%$ GP referrals were missed and 50\% locum referrals were inappropriate. There was confusion about whether to refer to paediatric urology or paediatrics $(40 \%$ and $30 \%$ respectively). $88 \%$ missed referrals related to atypical UTIs.

Conclusion NICE clinical guideline 54 is not easy to follow in a time pressured environment. This is evident across all grades of clinician. It was noted that patients presenting to out-of-hours or A\&E often do not have a urine sample sent for culture, hindering decisions regarding referral and further investigation. There was confusion about whether to refer to paediatrics or paediatric urology. Atypical UTIs were most likely to be mis-managed. An intuitive UTI flowchart has therefore been designed to facilitate easier identification of children who require tertiary referral and hence improve management.

\section{G306(P) RESEARCHAND PUBLIC AWARENESS PRIORITIES FOR SOUTH ASIAN CHILDREN, YOUNG PEOPLE AND THEIR FAMILIES: A COLLABORATIVE PARTICIPATORY APPROACH TO PRIORITISATION}

\begin{abstract}
${ }^{1,2} \mathrm{~L}$ Manikam, ${ }^{3} \mathrm{~K}$ Reed, ${ }^{2,4} \mathrm{G}$ Santini, ${ }^{5} \mathrm{R}$ Shah, ${ }^{1,2} \mathrm{M}$ Lakhanpaul. ${ }^{1}$ Population, Policy and Practice Programme, University College London Institute of Child Health, London, UK; ${ }^{2}$ Child Health Working Group, South Asian Health Foundation, Birmingham, UK; ${ }^{3}$ Kings College London School of Medicine, Guy's and St Thomas NHS Foundation Trust, London, UK; ${ }^{4}$ Independent Community Facilitator, Leicester, UK; ${ }^{5}$ Neonatal Unit, Homerton University Hospital NHS Foundation Trust, London, UK
\end{abstract}

\subsection{6/archdischild-2015-308599.283}

Aims To undertake a prioritisation exercise involving healthcare professionals (HCPs) and South Asian (SA) families to develop child health research and public awareness agendas'

Methods A two-stage process was adopted. A HCP scoping survey was undertaken to generate topics important for SA child health (1) research (2) public awareness and (3) outcome indicators. Ranked lists were discussed in four focus groups of SA adolescents and families.

A Punjabi and Urdu speaking community facilitator moderated groups with a British Sign Language interpreter assisting in the deaf group. Concordant and discordant themes between HCPs and SAs were identified.

Results 27 HCPs participated in the survey. Table 1 summarises their priorities

\begin{tabular}{ll} 
Abstract G306(P) Table 1 & Top HCP topics/outcome indicators \\
\hline Public Awareness & (1) Obesity and diet \\
(2) Mental health illness recognition \\
(3) Healthcare access and health seeking behaviour \\
(4) Vitamin D and rickets \\
(5) Routine health checks \\
(1) Nutrition, obesity and physical activity \\
(2) Diabetes \\
(3) Healthcare access and health seeking behaviour \\
(4) Health education \\
(5) Parent-child relationships and child care dynamics \\
(1) Growth, development and physical activity levels \\
(2) Health knowledge \\
(3) School attendance and literacy levels \\
(4) Healthcare utilisation \\
(5) Quality of life (QOL) scores
\end{tabular}

Abstract G306(P) Table 2 Topics prioritised/not prioritised by South Asians

\begin{tabular}{ll}
\hline Priorities & Not Priorities \\
(1) Concordance and shared decision making & (1) Genetic disorders and consanguinity \\
(2) Primary care access & (2) Diabetes \\
(3) Mental health & (3) Education/Literacy/School attendance \\
(4) Obesity and diet & (4) Parenting methods \\
(5) Blood and Organ donation & (5) QOL scores \\
(6) Alternative medicine effectiveness & \\
(7) Routine health monitoring &
\end{tabular}

35 individuals (Age range: 16-74 and UK stay length: 3-57 years) participated. Groups varied by settings (Inner vs. Outer city), religion, descent and disability.

Engagement was highest on public awareness and lowest on outcome indicators. Lack of awareness of research undertaken by funders (NIHR, Wellcome Trust, MRC) were cited. Table 2 summarises their priorities.

Conclusion Community engagement yielded research and public awareness priorities which differed with HCPs. In line with NHS England and NIHR national strategies, collaboration with communities whose views are not traditionally considered is essential to determine service and research agendas important to families, professionals and providers.

\section{G307(P) EVALUATING AND REDUCING PAEDIATRICS MEDICATION ERRORS BASED ON TWO AUDITS. "A MULTIDISCIPLINARY APPROACH"}

DC Atukorale, A Bhatti, R Jayatunga, A Ahmed. Paediatrics, Sandwell and West Birmingham Hospitals NHS Trust, West Bromwich, UK

\subsection{6/archdischild-2015-308599.284}

Aims Medication errors occur and are more significant in paediatrics, despite standards being set on safe prescribing (BNF \&Trust Prescribing Policy, 2007). Errors are frequent during prescribing, dispensing and administration of medications as shown by the EQUIP study (2009). We aimed to identify the incidence and types of medication errors and implement strategies to minimise these errors.

Method $1^{\text {st }}$ audit - was carried out to assess prescription charts against thirteen Good Prescribing standards (BNFC), in the inpatient unit.

$2^{\text {nd }}$ audit - A retrospective analysis was done of all incident reporting on paediatric medication errors within the Trust, over a 17 month period (January 2013-May 2014). Different types of medication errors, their location and the severity scoring was identified. The results were compared with a previous similar audit carried out in January 2011-May 2012, after which several interventions were implemented to reduce these errors.

Results Most of "Good Prescribing Practice"standards were met $(>80 \%)$, except for antibiotic indication and duration (Standard 13) $(20 \%)$.

Total Trust medication errors in January 2013-May 2014 were $10 \%$, out of which paediatric medication errors was $1 / 5^{\text {th }}$. Administration errors (47\%) dominated followed by prescription errors (42\%). 3\% were dispensing errors. Commonest administration error was failure to administer a prescribed medication and the commonest prescribing error was failure to prescribe a recommended medication. Errors on inpatient wards exceeded OPD/Community. 
Trust Incident Reporting Paediatric Medication Errors Serious Errors (amber)

$\begin{array}{llll}2011-2012 & 11.7 \% & 5.5 \% & 36 \% \\ 2013-2014 & 12.6 \% & 2.2 \% & 3 \%\end{array}$

Conclusions A significant reduction $(\mathrm{p}<0.05)$ in paediatric medication errors was seen after the multidisciplinary action plan was implemented, although the overall Trust incident reporting increased. Serious errors were significantly reduced ( $p$ $<0.001$ ) and there were no lethal paediatric medication errors during this period. The Root cause analysis and prescribing audit identified a number of interventions, including re-designing the Paediatric drug charts to include antibiotic indication and duration and the launch of the specials project to procure secondary care prescriptions in-house. We conclude that it is possible to reduce medication errors by implementing a multidisciplinary approach.

\section{G308(P) PARENTS THINK THAT 'MEDICINES FOR CHILDREN' REACHES NICE PLACES}

${ }^{1} \mathrm{M}$ Thomas, ${ }^{2} \mathrm{D}$ Tuthill. ${ }^{1}$ School of Medicine, Cardiff University, Cardiff, UK; ${ }^{2}$ Paediatrics, Children's Hospital for Wales, Cardiff, UK

\subsection{6/archdischild-2015-308599.285}

Background Whilst only $20 \%$ of content discussed in a consultation is retained, it's improved by giving additional written information. Patients are able to use written leaflets to consolidate their knowledge away from the stressful environment of a patient-doctor discussion. Such written information may increase treatment compliance and concordance. The 'Medicines for Children' website is designed to provide practical and reliable advice for families about giving medicine to their children. It has a variety of leaflets, videos and web-based information on over 200 children's medications. It's a partnership between the Royal College of Paediatrics and Child Health, the Neonatal and Paediatric Pharmacists Group and WellChild; a charity for parents and carers.

Objective To evaluate the 'Medicines for Children' website and information sheets, against the NICE quality standard 15; Understanding Treatment Options and NICE Medicines adherence guideline 76 ; involving patients in decision about prescribed medicines.

Methods A questionnaire was designed against the specific criteria set out in NICE quality standard 15 and clinical guideline 76. Questions focused on the layout, language and content of the leaflet, particularly information regarding the treatment risks and benefits. Data was collected from a convenience sample of parents attending children's outpatient clinics. Qualitative feedback was also sought.

Parents were asked to read the leaflet on 'Beclametasone inhaler for asthma prophylaxis' and answer the questionnaire as though their child were starting on this medication.

Results 106 parents participated. 16 declined.

\begin{tabular}{ll}
\hline Question & Percentage who agreed \\
\hline Good Leaflet Layout & $91 \%$ \\
Lay terminology used & $92 \%$ \\
Suitable content & $89 \%$ \\
Appropriate Information on medicine's benefits & $93 \%$ \\
Appropriate Information on medicine's risks & $93 \%$ \\
Would they use the Website in future? & $92 \%$ \\
\hline
\end{tabular}

Parents comments included: 'clearly laid-out with simple subheadings', 'written in a way that everyone can understand'. They suggested future developments should include: 'pictures for adults with lower literacy levels' and 'having the leaflets in both English and Welsh.'

Conclusion Medicines for Children information leaflets fulfil NICE standards and provide high quality information about children's medications which is highly valued by families.

\section{G309(P) "SAFETY HUDDLES": MULTIDISCIPLINARY VIEWS REGARDING THE PURPOSE AND EFFECTIVENESS OF A NOVEL PAEDIATRIC SITUATIONAL AWARENESS TOOL}

R Conn, J Adams, R Gohil. International and Private Patients Division, Great Ormond Street Hopsital NHS Trust, London, UK

\subsection{6/archdischild-2015-308599.286}

Aim Following an audit in 2012, which identified variability in the recognition and escalation of deteriorating patients, Safety Huddles were introduced, utilising a Childrens' Early Warning Score (CEWS), to enhance situation awareness.

Huddles are scheduled, regular multi-professional meetings, no longer than ten minutes, held in the clinical environment alongside an interactive electronic patient board. The sickest and most at risk patients (CEWs $>2$ ) are identified, prompting immediate and appropriate escalation. Four additional risk factors (family concerns, high risk therapies, clinicians' gut feeling and communication concerns) further identify patients as 'watchers.'

Huddles provide:

- Optimum safety through elimination of avoidable harm

- Greater empowerment and accountability of all staff through shared decision making

Our aim was to evaluate the attitudes and understanding of front-line staff regarding the purpose and effectiveness of Huddles, 18 months on.

Methods A voluntary, anonymous online survey was disseminated to staff across 3 clinical areas ( 2 wards, 1 outpatients).

Results 41 responses were returned. Respondents included 2 consultants, 4 registrars, 24 nurses, 1 nursing student, 1 healthcare assistant, 2 pharmacists and 5 interpreters.

The majority rated their understanding regarding the purpose of the Huddle as "good" (51\%) or "excellent" (41\%). $88 \%$ described the Huddle as an "important aspect" of their work, $98 \%$ no longer requiring reminders to attend. Subjectively, the Huddle led to improvements in: Team Communication (95\%), Patient/carer involvement (63\%), Staff support (80\%), identification of deteriorating patients (93\%) and timely escalation (90\%). $83 \%$ felt better informed about patients not specifically allocated to their care. $50 \%$ felt Huddles should occur with increased frequency.

Crucially, 93\% felt enabled to have their concerns heard.

Problems identified included punctuality of start times and occasional non-attendance of doctors, which subjectively lessened the value of the Huddle.

Conclusion Huddles are regarded as useful by the vast majority of staff and are an inclusive, empowering, non-hierarchical method of information sharing regarding patient safety. Our findings have been shared with all staff and suggested modifications are being considered.

Huddles are now being introduced across UK 12 sites as part of the SAFE collaborative of RCPCH. 